\title{
Suicide among Adult Psychiatric Inpatients: A Pilot Study in Iran
}

\author{
Saeed Shoja Shafti*, Alireza Memarie, Masomeh Rezaie and Masomeh Hamidi \\ Professor of Psychiatry, University of Social Welfare and Rehabilitation Sciences, Razi Psychiatric Hospital,Tehran, Iran \\ *Corresponding author: Saeed Shoja Shafti, Professor of Psychiatry, University of Social Welfare and Rehabilitation \\ Sciences, Razi Psychiatric Hospital,Tehran, Iran
}

\section{ARTICLE INFO}

Received: 彗 August 21, 2019

Published: 幽 August 27, 2019

Citation: Saeed Shoja S, Alireza M, Masomeh R, Masomeh H. Suicide among Adult Psychiatric Inpatients: A Pilot Study in Iran. Biomed J Sci \& Tech Res 20(5)-2019. BJSTR. MS.ID.003523.

Keywords: Psychiatric Disorders; Suicide; Suicide Attempt; First Admission; Recurrent Admission; Schizophrenia; Bipolar Disorder; Depression; Substance abuse Disorder

Abbreviations: FEP:FirstEpisodePsychosis; TG: Triglyceride; LDL: Low Density Lipoprotein; HDL High Density Lipoprotein

\section{ABSTRACT}

Introduction: Suicidal behavior is seen in the context of a variety of mental disorders and while many believe that, in general, first episode psychosis is a particularly highrisk period for suicide, no general agreement regarding higher prevalence of suicide in first episode psychosis is achievable. In the present study, suicides and suicide attempts among psychiatric in-patients has been evaluated to assess the general profile of suicidal behavior among native psychiatric inpatients and probing any relationship between serum cholesterol level and suicidal behavior.

Methods: Five acute academic wards, which have been specified for admission of first episode adult psychiatric patients, and five acute non-academic wards, which have been specified for admission of recurrent episode adult psychiatric patients, had been selected for current study. All inpatients with suicidal behavior (successful suicide and attempted suicide, in total), during the last five years (2013-2018), had been included in the present investigation. Also, assessment of serum lipids, including triglyceride, cholesterol, low density lipoprotein and high density lipoprotein, had been accomplished, for comparing the suicidal subjects with non-suicidal ones.

Results: Among 19160 psychiatric patients hospitalized in razi psychiatric hospital during a sixty months period, 63 suicidal behaviors, including one successful suicide and sixty two suicide attempts, had been recorded by the safety board of hospital. The most frequent mental illness was bipolar I disorder, which was significantly more prevalent in comparison with other mental disorders $(\mathrm{p}<0.04, \mathrm{p}<0.02, \mathrm{p}<0.007$, and $\mathrm{p}<0.003$ in comparison with schizophrenia, depression, personality disorders and substance abuse, respectively). Self-mutilation, self poisoning and hanging were the preferred methods of suicide among $61.11 \%, 19.44 \%$ and $19.44 \%$ of cases, respectively. In addition, no significant difference was evident between the first admission and recurrent admission inpatients, totally and separately, particularly with respect to psychotic disorders. Besides, with respect to different components of serum lipids, no specific or significant pattern was evident.

Conclusion: While in the present study the suicidal behavior was significantly more evident in bipolar disorder in comparison with other psychotic or no-psychotic disorders, no significant difference was evident between first admission and recurrent admission psychiatric inpatients. Moreover, no significant relationship between suicidal behavior and serum lipids was palpable.

\section{Introduction}

Suicide is derived from the Latin word for "self-murder." It is a fatal act that represents the person's wish to die. A suicide attempt is a behavior that the individual has undertaken with at least some intent to die. The behavior might or might not lead to death, injury or serious medical consequences. Several factors can influence the medical consequences of the suicide attempt, including poor planning, lack of knowledge about the lethality of the method chosen, low intentionality or ambivalence, or chance intervention by others after the behavior has been initiated [1] Determining the degree of intent can be challenging. Individuals might not acknowledge intent, especially in situations where doing 
so could result in hospitalization or cause distress to loved ones. Markers of risk include degree of planning, including selection of a time and place to minimize rescue or interruption; the individual's mental state at the time of the behavior, with acute agitation being especially concerning; recent discharge from inpatient care; or recent discontinuation of a mood stabilizer such as lithium or an antipsychotic such as clozapine in the case of schizophrenia.

Approximately 25\%-30\% of persons who attempt suicide will go on to make more attempts. Suicidal behavior is seen in the context of a variety of mental disorders, most commonly bipolar disorder, major depressive disorder, schizophrenia, schizoaffective disorder, anxiety disorders, substance use disorders, borderline personality disorder, antisocial personality disorder, eating disorders, and adjustment disorders. It is rarely manifested by individuals with no discernible pathology, unless in specific circumstances, like medical, political, or religious conflicts [1]. According to the findings of a study, among male psychiatric population, the absolute risk of suicide was highest for bipolar disorder, followed by unipolar affective disorder and schizophrenia. Among female psychiatric population, as well, the highest risk was found among women with schizophrenia, followed by bipolar disorder [2].

According to data, approximately 5\%-6\% of individuals with schizophrenia die by suicide, about $20 \%$ attempt suicide on one or more occasions, and many more have significant suicidal ideation. Suicidal behavior is sometimes in response to command hallucinations to harm oneself or others. Suicide risk remains high over the whole lifespan for males and females, although it may be especially high for younger males with comorbid substance use. Other risk factors include having depressive symptoms or feelings of hopelessness and being unemployed and the risk is higher, also, in the period after a psychotic episode or hospital discharge [3]. It is interesting that Bleuler had drawn clinicians' attention that the most serious of the schizophrenic symptoms is the suicidal drive [4]. Up to 50 percent of suicides among patients with schizophrenia occur during the first few weeks and months after discharge from a hospital; only a minority commit suicide while inpatients [3].

Having three or four hospitalizations during their 20s probably undermines the social, occupational, and sexual adjustment of possibly suicidal patients with schizophrenia. Consequently, potential suicide victims are likely to be male, unmarried, unemployed, socially isolated, and living alone-perhaps in a single room. After discharge from their last hospitalization, they may experience a new adversity or return to ongoing difficulties. As a result, they become dejected, experience feelings of helplessness and hopelessness, reach a depressed state, and have, and eventually act on, suicidal ideas [5]. Abrupt discontinuation of medication, poor treatment compliance, social isolation, and increased expectation of good performance from others and from patients themselves, are risk factors for suicide in schizophrenics [6]. Also, the lifetime risk of suicide in individuals with bipolar disorder is estimated to be at least 15 times that of the general population. In fact, bipolar disorder may account for one-quarter of all completed suicides [7].

Many believe that, in general, first episode psychosis (FEP) is a particularly high-risk period for suicide, in which risk elevates by $60 \%$ within a first year of treatment as compared to later stages of illness. In this regard, longer duration of untreated psychosis, greater symptoms of depression, and positive symptoms of psychosis were found to increase the odds of experiencing suicidal ideation [8]. While according to some studies depressive symptoms during the index psychotic episode and comorbidity with stimulant abuse at baseline were relevant predictive factors for suicidal behavior during the first years of first affective and non-affective psychotic episodes [9], more depressive symptoms, higher insight, and negative beliefs about psychosis increase the risk for suicidality in FEP [10]. Impulsive behavior such as self-harm, as well as having a family history of severe mental disorder or substance use, have been stated as important risk factors for suicide in FEP $[11,12]$.

Furthermore, low levels of cholesterol have been described in suicide behavior including among those individuals who have an increased tendency for impulsivity $[13,14]$. While, as a kind of psychological explanation, some scholars believe that young men in the early stages of their treatment are seeking to find meaning for frightening, intrusive experiences with origins which often precede psychosis, and these experiences invade personal identity, interactions and recovery [15], some suggests that personality characters, specifically, passive-dependent traits can be a predictor of first suicide attempts FEP [16]. On the other hand, no general agreement regarding higher prevalence of suicide in FEP is so far achievable. For example, while researchers like Nordentoft et al. $[2,8,17,18]$. have stated that FEP is a particularly high-risk period for suicide, with a risk as high as $10-60 \%$ during the first year of treatment, other scholars like Preti et al. [19-22], have expected a lower risk or stated that suicide rates are difficult to measure in FEP patients, even in carefully defined samples. In the present study, suicides and suicide attempts among psychiatric in-patients, during the last five years, in Razi psychiatric hospital, as the largest national psychiatric hospital in Iran and region, has been evaluated to assess the general profile of suicidal behavior among native psychiatric inpatients, comparing first admission with recurrent admission patients, and probing any relationship between serum cholesterol level and suicidal behavior.

\section{Methods}

Razi psychiatric hospital, as the largest psychiatric hospital in the middle east, which is located in south of capital city of Tehran, with a capacity around 1375 active beds, had been selected as the field of study in the present assessment. Amongst its separate existent sections, five acute academic wards, which have been specified for admission of first episode adult psychiatric patients, and five acute non-academic wards, which have been specified for admission of recurrent episode adult psychiatric patients, with a 
collective capacity around two hundred active beds in each cluster ( four hundreds beds, totally), had been selected for current study. Among the aforesaid academic divisions, two wards included female inpatients, with around eighty beds, and the remaining three wards included male inpatients. All non-academic wards involved male inpatients. For valuation, all inpatients with suicidal behavior (successful suicide and attempted suicide, in total), during the last sixty months, had been included in the present investigation. Besides, clinical diagnosis was based on Diagnostic and Statistical Manual of Mental Disorders, $5^{\text {th }}$ edition (DSM-5) (23). Also, assessment of serum lipids, including triglyceride (TG), cholesterol, low density lipoprotein (LDL) and high density lipoprotein (HDL), which was part of routine laboratory checkups for all patients upon admission, whether for the first time or periodically, had been accomplished, for comparing the suicidal subjects with nonsuicidal ones, incidentally.

\section{Statistical Analyses}

While 't-test' has been used for comparison of means as regards mean total level of serum lipids, difference of suicidal behavior between first admission and recurrent admission patients, had been analyzed by 'comparison of proportions'. Statistical significance as well, had been defined as p value $\leq 0.05$. MedCalc Statistical Software version 15.2 was used as statistical software tool for analysis.

\section{Results}

As said by the results, among 19160 psychiatric patients hospitalized in razi psychiatric hospital, during a sixty months period (2013-2018), sixty three suicidal behaviors, including one successful suicide and sixty two suicide attempts, had been recorded by the security board of hospital (Table 1). Thirty three of patients were male and thirty of them were female, with no significant difference with regard to quantity (Table 2). The most frequent mental illness was bipolar I disorder (34.92\%), which was significantly more prevalent in comparison with other mental disorders ( $\mathrm{p}<0.04, \mathrm{p}<0.02, \mathrm{p}<0.007$, and $\mathrm{p}<0.003$ in comparison with schizophrenia, depression, personality disorders and substance abuse, respectively). The other disorders included schizophrenia (19.04\%), major depressive disorder (MDD) (17.46\%), personality disorders (borderline \& antisocial) (14.28\%), substance abuse disorders, especially methamphetamine induced psychosis (MIP) (12.69\%), and adjustment disorder (1.58\%) (Table 3) (Figure1). Also, no significant difference was evident between the first admission and recurrent admission inpatients, totally $(\mathrm{p}<0.31)$ and separately, particularly with respect to psychotic disorders (Table 3) (Figure 2). The annual incidences of suicidal behavior in both groups were comparable, and they were around $0.035 \%$ and $0.030 \%$, in first admission and recurrent admission psychiatric inpatients, respectively (Table 1). While self-mutilation, self poisoning and hanging were the preferred methods of suicide among $61.11 \%, 19.44 \%$ and $19.44 \%$ of cases, respectively, the first style was significantly more prevalent than the other ways ( $\mathrm{Z}=1.96, \mathrm{P}<0.059$, CI: $-0.0088,0.4532$ ). Furthermore, no significant gender-based difference was evident with respect to the style of suicide in the present assessment (Figure 3). Besides, with respect to different components of serum lipids, no specific or significant pattern was evident, except that all hypolipidemic patients $(n=7)$ were diagnosed as major depressive disorder, while $80 \%$ of hyperlipidemic patients $(\mathrm{n}=5)$ were diagnosed as bipolar I disorder (Table 4).

Table 1: Comparing suicidal behavior between first admission and recurrent admission psychiatric patients in Razi psychiatric hospital thru 2013-2018.

\begin{tabular}{|c|c|c|c|c|c|c|c|c|c|}
\hline $\begin{array}{c}\text { Total } \\
\text { number of } \\
\text { patients }\end{array}$ & $\begin{array}{c}\text { First } \\
\text { admissions }\end{array}$ & $\%$ & $\begin{array}{c}\text { Annual } \\
\text { Incidence }\end{array}$ & $\begin{array}{c}\text { Recurrent } \\
\text { admissions }\end{array}$ & \% & $\begin{array}{c}\text { Annual } \\
\text { Incidence }\end{array}$ & Z & P & CI \\
\hline 19160 & $\mathrm{~N}=34$ & 0.17 & 0.035 & $\mathrm{~N}=29$ & 0.15 & 0.030 & 0.890 & 0.373 & $-0.09,0.25$ \\
\hline
\end{tabular}

Table 2: Gender difference in suicidal behavior.

\begin{tabular}{|c|c|c|c|c|c|}
\hline Suicidal cases & Male & Female & z & P & CI \\
\hline $\mathrm{N}=63$ & 33 & 30 & 0.53 & 0.59 & $-0.12,0.22$ \\
\hline
\end{tabular}

Table 3: Frequency of psychiatric disorders among suicidal patients.

\begin{tabular}{|c|c|c|c|c|c|}
\hline Psychiatric disorders & First admission & Recurrent admission & z & P & CI \\
\hline Major depressive disorder & 5 & 6 & -0.42 & 0.66 & $-0.50,0.32$ \\
\hline Bipolar I disorder & 10 & 12 & 0.60 & 0.54 & $-0.20,0.38$ \\
\hline Schizophrenia & 8 & 4 & 1.63 & 0.10 & $-0.06,0.73$ \\
\hline Substance abuse & 5 & 3 & 1.00 & 0.31 & $-0.24,0.74$ \\
\hline Personality disorders & & & & & \\
\hline (borderline \& antisocial) & 5 & 4 & 0.47 & 0.63 & $-0.35,0.57$ \\
\hline Adjustment disorder & 1 & 0 & 1.41 & 0.15 & $-0.38,2.38$ \\
\hline Total & 34 & 29 & 0.89 & 0.37 & $-0.09,0.25$ \\
\hline
\end{tabular}


Table 4: Lipid profile of suicidal patients.

\begin{tabular}{|c|c|c|c|}
\hline Serum Lipids & $\begin{array}{c}\text { Mean total levels in normal } \\
\text { patients } \mathbf{N = 5 1}\end{array}$ & $\begin{array}{c}\text { Mean total levels in } \\
\text { Hyperlipidemic patients } \\
\mathbf{N = 5} \mathbf{( 2 0 \%} \text { MDD; 80\% BID) }\end{array}$ & $\begin{array}{c}\text { Hypolipidemic patients } \\
\text { N=7 (100\% MDD) }\end{array}$ \\
\hline Cholesterol & $164.6 \pm 18.66$ & $135.33 \pm 22.95$ & $135.75 \pm 20.75$ \\
\hline Triglyceride & $129.6 \pm 36.67$ & $164.66 \pm 23.57$ & $83.75 \pm 27.22$ \\
\hline LDL & $96 \pm 9.27$ & $51.66 \pm 13.67$ \\
\hline HDL & $46.4 \pm 16.71$ & $34.25 \pm 15.12$ & \\
\hline
\end{tabular}

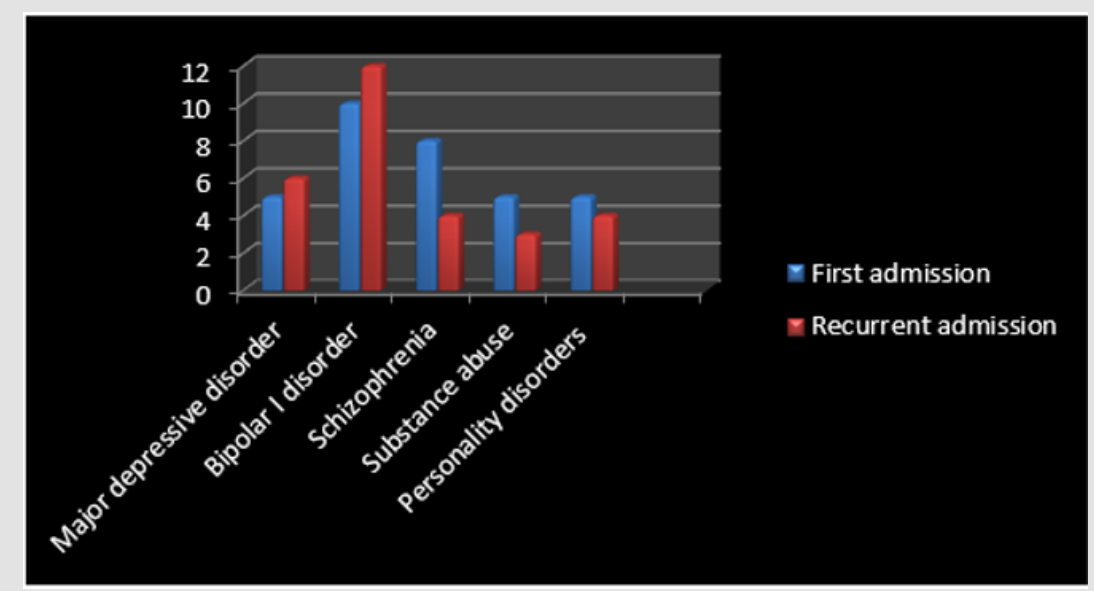

Figure 1: Diagrammatic representation of psychiatric disorders among suicidal subjects.

Figure 2: Graphic illustration of first and recurrent admissions' suicidal behavior.

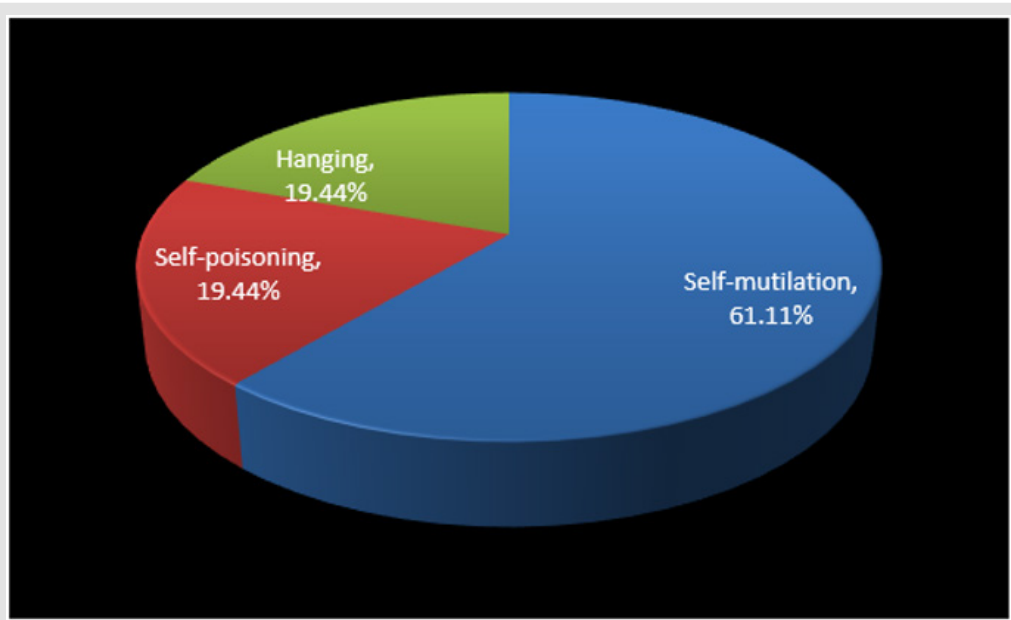

Figure 3: Prevalence of different styles of suicide among inpatients. 


\section{Discussion}

Always in psychiatry, when giving information about the diagnosis, course of illness, and treatment, the therapist should not ignore the risk of suicide [1]. Also, there is a high proportion of young people with first-episode psychosis who attempted suicide before their first contact with mental health services. This finding suggests that the mortality rates associated with psychotic disorders may be underreported because of suicide deaths taking place before first treatment contact [24]. It should constantly be considered that in the psychiatric hospital setting the inpatient at risk for suicide has previously exhibited suicidal behavior, suffers from schizophrenia, was admitted involuntarily, and lives alone [24]. It is interesting that among persons hospitalized, the risk of suicide was greater in 1985-1991 than in 1995-2001 for post discharge period, particularly for patients with schizophrenia and patients with affective disorders. Thus, not only the restructuring and downsizing of mental health services was not associated with any increase in suicides, the risk of suicides decreased significantly between the two time periods among several diagnostic categories.

But, while in terms of post-discharge suicides, the downsizing of psychiatric hospitals has been a success, there is still a substantial need for better recognition of suicidal risk among psychiatric patients [25]. According to a survey, there are 2 sharp peaks of risk for suicide around psychiatric hospitalization, one in the first week after admission and another in the first week after discharge; suicide risk is significantly higher in patients who received less than the median duration of hospital treatment; affective disorders have the strongest impact on suicide risk in terms of its effect size and population attributable risk; and suicide risk associated with affective and schizophrenia spectrum disorders declines quickly after treatment and recovery, while the risk associated with substance abuse disorders declines relatively slower [26]. The accessibility to one or more means of suicide is a recognized factor in psychiatric institutions. The same is true for the conditions of care: inadequate supervision, the underestimation of the risk of suicide by teams, poor communication within the teams and the lack of intensive care unit promote suicide risk [27]. But according to another study in FEP, the majority of attempts occurred when patients were treated as outpatients and were in regular contact with the service [17]. As suicide is a relatively rare event in psychotic disorders, general population-based prevention strategies may have more impact in this vulnerable group as well as the wider population $[28,29]$.

While the immediate post-discharge period is a time of marked risk, rates of suicide remain high for many years after discharge and patients admitted because of suicidal ideas or behaviors and those in the first months after discharge should be a particular focus of concern [30]. Back to our discussion and according to the findings of the present study, The most common principal diagnoses among the suicide subjects were bipolar I disorder and schizophrenia, which was similar to the findings of Nordentoft et al.[2], except that no gender difference regarding prevalence of these disorders among male and female patients was repeatable here. But, our findings were not in complete agreement with the conclusion of Thong JY et al., who had found only schizophrenia and depression as the most common principal diagnoses among their suicide subjects [31] and Roy et al., who had declared schizophrenia as the major diagnosis among suicide victims [24]. Meanwhile other psychiatric disorders, like personality disorders and substance abuse disorders, in addition to the aforementioned diagnoses, had been designated in the present study, as remarkable diagnoses among suicide subjects; though fewer than the aforementioned diagnoses.

The higher prevalence of bipolar disorder in the present assessment is likewise comparable to the outcomes of a further study regarding suicidal behavior among Iranian inpatient youngsters [32]. In the same way, the higher incidence of self-mutilation, as the preferred method of suicide in the present evaluation, was parallel to the said study, except than it's significantly higher prevalence amongst female adolescents [33]. Also, in keeping with the results, while the annual incidences of suicidal behavior in both groups were comparable, they were lower than assessments of Björkenstam et al. [11,34] and higher than approximations of Li et al. [35], which could be stemmed from cultural, instrumental, diagnostic and methodical differences. Also, in accordance with the outcomes of the present assessment, no significant difference was evident between the first admission and recurrent admission inpatients, totally and separately, particularly with respect to psychotic disorders.

Such an outcome is clearly incongruous with the findings of Nordentoft et al. $[2,8,17,18]$, who have stated that first episode psychosis is a particularly high-risk period for suicide and firstepisode psychotic disorder, in general, has seemed to be a highrisk population for suicidal behavior during the first year of treatment. On the other hand, our findings are compatible with the stances of Preti et al. [19-22], who have estimated a lower risk or indicated that suicide rates are difficult to measure in FEP patients, and there is relatively little specific information about the risk of suicide at illness onset or retrospectively concerning the untreated psychotic period. Above and beyond, with respect to relationship between serum lipids and suicidal behavior, outcome of the present assessment was not in harmony with the findings of Shrivastava et al. $[13,14]$ because while there was a couple of patients with higher or lower serum level of cholesterol, triglyceride, LDL and HD, no specific or significant pattern was evident in this regard; so, it seems that maybe such a difference was associated more to alteration of appetite, as a secondary phenomenon, rather than core variation of metabolism, as a primary etiologic issue. Anyhow, disregard to outcomes of the present study and its similarities or differences with comparable studies, elements of an inclusive prevention policy can be grouped under five items: securing the hospital environment, optimization of the care of the patients at suicidal risk, training of the medical teams in the detection of the 
risk and in the care of the suicidal subjects, involvement of the families in the care and implementation of post-event procedures following a completed suicide or an attempt [36].

Also, to reduce the number of suicide attempts among individuals treated for FEP, psychiatric services could consider: restricting the amount of medication prescribed per purchase; individualized suicide risk management plans for all newly admitted patients, including those who do not appear to be at risk; stringent reviews of inpatient psychiatric units for potential ligature points; providing information and psycho-education for significant others in recognition and response to suicide risk; fostering patients' problem solving and conflict resolution skills; and regular risk assessment and close monitoring of patients, particularly during the high risk period of 3 months after a suicide attempt [17]. Also, along with enhancement of insight, coping strategies should be boosted with a goal of minimizing depression and preventing suicidality [37]. Absence of post- discharge following program, deficiency of documented data regarding the suicidal behavior or its idea before admission, allocating wards to academic and non-academic, which could impact the quality of care, lack of female gender in the nonacademic wards for making it more similar to the academic wards, which included an equal mixture of male and female inpatients, were among the weaknesses of the present assessment. In spite of remarkable findings of the current study, more methodical and comprehensive investigations in future, with taking into account the above shortages, can improve the quality and amendment of mental health services for proper response to patients' unavoidable problems.

\section{Conclusion}

While in the present study the suicidal behavior was significantly more evident in bipolar disorder in comparison with other psychotic or no-psychotic disorders, no significant difference was evident between first admission and recurrent admission psychiatric inpatients. Moreover, no significant relationship between suicidal behavior and serum lipids was palpable.

\section{References}

1. (2013) American Psychiatric Association (APA). Diagnostic and statistical manual of mental disorders. ( $5^{\text {th }}$ edn). Washington (DC): American Psychiatric Association pp. 801-806.

2. Nordentoft M, Madsen T, Fedyszyn I (2015) Suicidal behavior and mortality in first-episode psychosis. J Nerv Ment Dis 203(5): 387-392.

3. (2013) American Psychiatric Association (APA). Diagnostic and statistical manual of mental disorders. ( $5^{\text {th }}$ edn.).Washington (DC): American Psychiatric Association pp. 104.

4. Kahn MW (2001) Emergency psychiatry: tools of engagement: avoiding pitfalls in collaborating with patients. Psychiatr Serv 52(12): 1571-1572.

5. Ballard ED, Stanley Ill, Horowitz LM, Cannon EA, Pao M, et al. (2013) Asking youth questions about suicide risk in the pediatric emergency department: Results from a qualitative analysis of patient opinions. Clin Pediatr Emerg Med 14(1): 20-27.

6. Hillard R, Zitek B (2004) Emergency Psychiatry. New York, NY: McGrawHill Companies.
7. (2013) American Psychiatric Association (APA). Diagnostic and statistical manual of mental disorders. 5th ed. Washington (DC): American Psychiatric Association p. 131.

8. Bornheimer LA (2018) Suicidal Ideation in First-Episode Psychosis (FEP): Examination of Symptoms of Depression and Psychosis Among Individuals in an Early Phase of Treatment. Suicide Life Threat Behav 23(2): 45-47.

9. González Pinto A, Aldama A, González C, Mosquera F, Arrasate M, et al. (2007) Predictors of suicide in first-episode affective and nonaffective psychotic inpatients: five-year follow-up of patients from a catchment area in Vitoria, Spain. J Clin Psychiatry 68(2): 242-247.

10. Barrett EA, Sundet K, Faerden A, Agartz I, Bratlien U, et al. (2010) Suicidality in first episode psychosis is associated with insight and negative beliefs about psychosis. Schizophr Res 123(2-3): 257-262.

11. Björkenstam C, Björkenstam E, Hjern A, Bodén R, Reutfors J (2014) Suicide in first episode psychosis: a nationwide cohort study. Schizophr Res 157(1-3): 1-7.

12. Beckman K, Mittendorfer Rutz E, Lichtenstein P, Larsson H, Almqvist C, et al. (2016) Mental illness and suicide after self-harm among young adults: long-term follow-up of self-harm patients, admitted to hospital care, in a national cohort. Psychol Med 46(16): 3397-3405.

13. Shrivastava A, Johnston M, Campbell R, De Sousa A, Shah N (2017) Serum cholesterol and Suicide in first episode psychosis: A preliminary study. Indian J Psychiatry 59(4): 478-482.

14. Ayesa Arriola R, Canal Rivero M, Delgado Alvarado M, Setién Suero E, González Gómez J, et al. (2018) Low-density lipoprotein cholesterol and suicidal behaviour in a large sample of first-episode psychosis patients. World J Biol Psychiatry 19: 158-161.

15. Gajwani R, Larkin M, Jackson C (2018) What is the point of life? : An interpretative phenomenological analysis of suicide in young men with first-episode psychosis. Early Interv Psychiatry 12(6): 1120-1127.

16. Canal Rivero M, Barrigón ML, Perona Garcelán S, Rodriguez Testal JF, Giner L, et al. (2016) One-year follow-up study of first suicide attempts in first episode psychosis: Personality traits and temporal pattern. Compr Psychiatry 71: 121-129.

17. Fedyszyn IE, Harris MG, Robinson J, Edwards J, Paxton SJ (2011) Characteristics of suicide attempts in young people undergoing treatment for first episode psychosis. Aust N Z J Psychiatry 45(10): 838845 .

18. Cohen S, Lavelle J, Rich CL, Bromet E (1994) Rates and correlates of suicide attempts in first-admission psychotic patients. Acta Psychiatr Scand 90(3): 167-171.

19. Preti A, Meneghelli A, Pisano A, Cocchi A (2009) Risk of suicide and suicidal ideation in psychosis: results from an Italian multi-modal pilot program on early intervention in psychosis. Schizophr Res 113(2-3): 145-150.

20. Pompili M, Serafini G, Innamorati M, Lester D, Shrivastava A, et al. (2011) Suicide risk in first episode psychosis: a selective review of the current literature. Schizophr Res 129(1): 1-11.

21. Crumlish N, Whitty P, Kamali M, Clarke M, Browne S, et al. (2005) Early insight predicts depression and attempted suicide after 4 years in firstepisode schizophrenia and schizophreniform disorder. Acta Psychiatr Scand 112(6): 449-455.

22. Addington J, Williams J, Young J, Addington D (2004) Suicidal behaviour in early psychosis. Acta Psychiatr Scand 109(2): 116-120.

23. (2013) American Psychiatric Association (APA). Diagnostic and Statistical Manual of Mental Disorders. ( $5^{\text {th }}$ edn.). Washington, DC: American Psychiatric Association pp. 31-715.

24. Roy A, Draper R (1995) Suicide among psychiatric hospital in-patients. Psychol Med 25(1): 199-202.

25. Pirkola S, Sohlman B, Heilä H, Wahlbeck K (2007) Reductions in postdischarge suicide after deinstitutionalization and decentralization: a nationwide register study in Finland. Psychiatr Serv 58(2): 221-226. 
26. Qin P, Nordentoft M (2005) Suicide risk in relation to psychiatric hospitalization: evidence based on longitudinal registers. Arch Gen Psychiatry 62(4): 427-432.

27. Martelli C, Awad H, Hardy P (2010) In-patients suicide: epidemiology and prevention. Encephale. 36(2): 83-91.

28. Dutta R, Murray RM, Allardyce J, Jones PB, Boydell J (2011) Early risk factors for suicide in an epidemiological first episode psychosis cohort. Schizophr Res 126(1-3): 11-19.

29. Dutta R, Murray RM, Hotopf M, Allardyce J, Jones PB, et al. (2010) Reassessing the long-term risk of suicide after a first episode of psychosis. Arch Gen Psychiatry 67(12): 1230-1237.

30. Chung DT, Ryan CJ, Hadzi Pavlovic D, Singh SP, Stanton C, et al. (2017) Suicide Rates After Discharge From Psychiatric Facilities: A Systematic Review and Meta-analysis. JAMA Psychiatry 74(7): 694-702.

31. Thong JY, Su AH, Chan YH, Chia BH (2008) Suicide in psychiatric patients: case-control study in Singapore. Aust N Z J Psychiatry 42(6): 509-519.

ISSN: 2574-1241

DOI: 10.26717/BJSTR.2019.20.003523

Saeed Shoja Shafti. Biomed J Sci \& Tech Res

(C) (P) This work is licensed under Creative

Submission Link: https://biomedres.us/submit-manuscript.php
32. Shoja Shafti S, Memarie A, Rezaie M, Hamidi M (2019) Clinical Profile of Suicide among Adolescent Psychiatric Inpatients in Iran. Biomedical journal of Scientific \& Technical Research 18(1): 13242-13246.

33. Shoja Shafti S, Memarie A, Rezaie M, Hamidi M (2019) Suicidal Conduct among Youthful Psychiatric Inpatients: A pilot Local Survey. Peer Reviewed Journal of Forensic \& Genetic sciences 3(4): 216-220.

34. Neuner T, Schmid R, Wolfersdorf M, Spiessl H (2008) Predicting inpatient suicides and suicide attempts by using clinical routine data? Gen Hosp Psychiatry 30(4): 324-330.

35. Li J, Ran MS, Hao Y, Zhao Z, Guo Y, et al. (2008) Inpatient suicide in a Chinese psychiatric hospital. Suicide Life Threat Behav 38(4): 449-455.

36. Martelli C, Awad H, Hardy P (2010) In-patients suicide: epidemiology and prevention. Encephale 36 (2): 83-91.

37. Flanagan P, Compton MT (2012) A comparison of correlates of suicidal ideation prior to initial hospitalization for first-episode psychosis with prior research on correlates of suicide attempts prior to initial treatment seeking. Early Interv Psychiatry 6(2): 138-144.

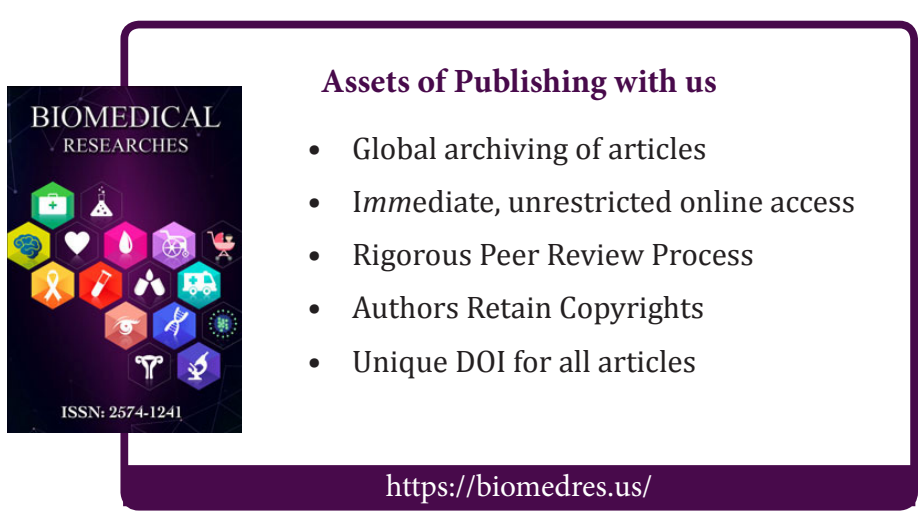

